\title{
FUNÇÕES E DISFUNÇÕES PÉLVICAS: PAPEL DA FISIOTERAPIA PÓS CIRURGIA DE AFIRMAÇÃO DE GÊNERO EM MULHERES TRANSEXUAIS
}

\author{
Roberta Paganini, ' Adriana Nagahama, ${ }^{2}$ Fernanda Antico Benetti, ${ }^{3}$ Amanda Estevão, ${ }^{4}$ Mariane Castiglione ${ }^{5}$ \\ PELVIC FUNCTIONS AND DYSFUNCTIONS: ROLE OF PHYSIOTHERAPY AFTER GENDER \\ AFFIRMATION SURGERY IN TRANSEXUAL WOMEN
}

\section{FUNCIONES Y DISFUNCIONES PÉLVICAS: PAPEL DE LA FISIOTERAPIA ANTE LA CIRUGÍA DE AFIRMACIÓN DE GÉNERO EN MUJERES TRANSEXUALES}

\begin{abstract}
Resumo: a cirurgia de afirmação de gênero é um procedimento cujo objetivo é estabelecer as pessoas transexuais a completa interação entre o corpo, a mente e a identidade sexual. Assim como outras cirurgias existem diversas complicações que podem ocorrer, entretanto, são pouco elucidadas na literatura, igualmente a atuação da fisioterapia frente a essas complicações. Objetivo: analisar as funções e disfunções pélvicas, e verificar a atuação da fisioterapia pós cirurgia de afirmação de gênero em mulheres transexuais. Metodologia: trata-se de uma revisão de literatura integrativa. Para execução foram realizadas buscas nas bases de dados PEDro e PubMed. Os descritores em ciências da saúde utilizados foram: "Pessoas Transgênero", "Modalidades de Fisioterapia”, "Assoalho Pélvico" e "Transexualismo". Resultados: foram encontrados nove artigos que contemplavam a proposta idealizada. Foi observado que existe consenso em relação à alta incidência de complicações pélvicas, entretanto, a atuação da fisioterapia foi citada apenas em dois estudos. A função sexual, é um dado conflitante, visto que algumas pacientes relatam incapacidade de realizar qualquer ato sexual, e outras relatam orgasmos frequentes; em relação a funcionalidade, estética e qualidade de vida as pacientes apresentam boa satisfação. Conclusão: a cirurgia de afirmação de gênero está relacionada a complicações pós-operatórias relevantes e podem impactar na função sexual, entretanto, as pacientes apresentam melhora da qualidade de vida após o procedimento, o que pode estar relacionado a fatores psíquicos e físicos. A atuação da fisioterapia pode ser promissora nesse âmbito, entretanto é pouco elucidada na literatura cientifica.
\end{abstract}

Palavras-chave: pessoas transgênero; modalidades de fisioterapia; assoalho pélvico; transexualismo.

Abstract: Gender affirmation surgery is a procedure whose goal is to establish transsexual people the complete interaction between the body, mind and sexual identity. Like other surgeries, there are several complications that may occur, however, they are little elucidated in the literature, also the performance of physiotherapy in the face of these complications. Objective: To analyze pelvic functions and dysfunctions, and verify the performance of physiotherapy after gender affirmation surgery in transsexual women. Methodology: This is an integrative literature review. For execution, searches were performed in the PEDro and PubMed databases. The descriptors in health sciences used were: "Transgender persons", "Physical Therapy Modalities", "Pelvic Floor" and "Transexualism". Results: Nine articles were found that contemplate the idealized proposal. It was observed that there is consensus regarding the high incidence of pelvic complications, however, the performance of physiotherapy was mentioned only two studies. Sexual function is a conflicting data, since some patients report inability to perform any sexual act, and others report frequent orgasms; in relation to functionality, aesthetics and quality of life patients have good satisfaction. Conclusion: Gender affirmation surgery is related to relevant postoperative complications and may impact sexual function, however, patients present

\footnotetext{
I Discente do curso de Fisioterapia, Centro Universitário Saúde ABC. E-mail: roberta.paganini@hotmail.com

2 Discente do curso de Fisioterapia, Centro Universitário Saúde ABC. E-mail: adri.nagahama@gmail.com

${ }_{3}^{3}$ Fisioterapeuta e docente, doutora, Centro Universitário Saúde ABC. E-mail: benetti.fernanda@gmail.com

${ }^{4}$ Fisioterapeuta e docente, especializada, Centro Universitário Saúde ABC. E-mail: manda.est@hotmail.com

${ }^{5}$ Fisioterapeuta e docente, especializada, Centro Universitário Saúde ABC. E-mail: mariane.castiglione@fmabc.br
} 
improvement in quality of life after the procedure, which may be related to psychological and physical factors. The performance of physiotherapy may be promising in this context, however it is little elucidated in the scientific literature.

Keywords: transgender people; physiotherapy modalities; pelvic floor; transsexualism.

Resumen: La cirugía de afirmación de género es un procedimiento cuyo objetivo es establecer a las personas transexuales en completa interacción entre el cuerpo, la mente y la identidad sexual. Al igual que ocurre con otras cirugías, existen varias complicaciones que pueden presentarse, sin embargo, están poco esclarecidas en la literatura, como es el papel de la fisioterapia ante estas complicaciones. Objetivo: Analizar las funciones y disfunciones pélvicas y verificar el papel de la fisioterapia después de la cirugía de afirmación de género en mujeres transexuales. Metodología: Se trata de una revisión bibliográfica integradora. Las búsquedas se realizaron en las bases de datos PEDro y PubMed. Los descriptores en ciencias de la salud utilizados fueron: "Personas Trans", "Modalidades de Fisioterapia”, "Suelo Pélvico" y "Transexualismo". Resultados: Se encontraron nueve artículos que contemplaban la propuesta idealizada. Se observó que existe consenso en cuanto a la alta incidencia de complicaciones pélvicas, sin embargo, el papel de la fisioterapia se mencionó solo en dos estudios. La función sexual es un dato contradictorio, ya que algunos pacientes refieren una incapacidad para realizar cualquier acto sexual, y otros refieren orgasmos frecuentes y en relación a la funcionalidad, estética y calidad de vida, los pacientes tienen buena satisfacción. Conclusión: La cirugía de afirmación de género está relacionada con complicaciones posoperatorias relevantes y puede afectar la función sexual, sin embargo, los pacientes tienen una mejor calidad de vida después del procedimiento, lo que puede estar relacionado con factores psicológicos y físicos. El papel de la fisioterapia puede ser prometedor en este contexto, sin embargo, está poco aclarado en la literatura científica.

Palabras clave: personas trans; modalidades de fisioterapia; suelo pélvico; transexualidad.

\section{Introdução}

A sexualidade humana não é uma função apenas biológica que se restringe à genitalidade, mas à corporalidade como um todo. É um fenômeno que, além de envolver os aspectos biológicos como a anatomia e a fisiologia do sexo, inclui os aspectos psicológicos, sociológicos, religiosos, educacionais, físicos e ambientais. Não havendo uma supremacia entre eles. Assim, são desenvolvidos em um processo contínuo que se inicia antes mesmo do nascimento encerrando-se com a morte (VIEIRA et al., 20I7).

A sexualidade é responsável pela formação social do indivíduo, pois a identidade de gênero e a orientação sexual estão amplamente correlacionadas (VENTRIGLIO; BHUGRA, 2019). Atualmente, a definição de identidade de gênero é

O sentimento inerente e profundo de uma pessoa de ser menino, menina, homem, mulher, ou um gênero alternativo que pode, ou não, corresponder ao sexo atribuído ao nascimento ou às características sexuais primárias e secundárias de uma pessoa (AMERICAN PSYCHOLOGICAL ASSOCIATION, 20I5, p. 834).

A orientação sexual, é a capacidade de cada pessoa experimentar uma profunda atração emocional, afetiva ou sexual por indivíduos de gênero diferente, do mesmo gênero ou de mais de um gênero (JESUS, 20I2).
Transgênero é a pessoa que não se identifica com o gênero designado ao nascimento, um termo que engloba várias identidades, como homens e mulheres transsexuais, pessoas não binárias, travesti e outras (CIASCA; HERCOWITZ; LOPES, 202I).

Os indivíduos transexuais, cuja identidades, expressões ou comportamentos de gênero diferem daqueles previstos por seu sexo atribuído ao nascimento (JOSEPH et al., 20I7), ou seja, os indivíduos que não se identificam com o seu corpo e com o gênero que lhe foi designado ao nascimento, são definidos como transexuais, englobam aqueles que não se sentem afiliados ao sexo que the foi compulsoriamente designado e esperam adequar a sua imagem corpórea ao gênero ao qual se identificam. Entre pessoas transexuais, algumas optam por realizar a cirurgia de redesignação sexual ou de afirmação de gênero, e/ou passam pelo processo de hormonização (PORTO, 2020).

No que diz respeito à cirurgia, a redesignação sexual (CRS) ou afirmação de gênero, é o procedimento específico para pessoas diagnosticadas com disforia de gênero, cujo objetivo é estabelecer aos transexuais a completa interação entre corpo, mente e identidade sexual (ROCON et al., 2016).

A redesignação sexual de masculino para feminino é um método cirúrgico realizado para a reafirmação de gênero caracterizado por diferentes métodos cirúrgicos, porém a mais utilizada é a orquiectomia, que consiste na retirada dos testículos, criação da cavidade da neovagina, 
vaginoplastia, revestimento da cavidade, reconstrução do hiato uretral, construção dos pequenos lábios e grandes lábios, além da formação clitóris (FERREIRA; SILVA, 2020). Assim, como todas as cirurgias, há diversas complicações que podem ocorrer como consequência da CRS. Entre elas estão a estenose vaginal e uretral, prolapso vaginal, disfunções sexuais e fraqueza da parede vaginal (FERREIRA; CAMPOS; FERREIRA, 2018). Além destas, são citadas fístulas, retenções urinárias, sangramentos, infecções, lesões retais e necroses teciduais (SOUSA et al., 2019).

O papel da fisioterapia ocorre através de recursos direcionados ao tratamento das disfunções do assoalho pélvico, criam-se propostas terapêuticas com o objetivo de melhorar a adequação da musculatura pélvica à sua nova inserção e à nova função; orientações e exercícios para manutenção do canal vaginal, dessensibilização e adequação sensorial, promoção do incremento funcional desta nova inserção muscular ou minimização das queixas relacionadas às consequências do pós-operatório (FERREIRA; SILVA, 2020). Dentre as técnicas fisioterapêuticas mais utilizadas, estão a massagem perineal, eletroestimulação transcutânea do nervo tibial posterior, cinesioterapia, biofeedback pressórico e orientações, dependendo da queixa referida pela paciente (GALVÃO et al., 20I8).

Apesar de o Brasil obter um sistema de saúde igualitário e universal a todos os níveis de assistência de acordo com os princípios e diretrizes do SUS da Lei $n^{\circ} 8.080$, a realidade que esse grupo social enfrenta não é tão simples. No que diz respeito à saúde, o público trans tem inúmeras dificuldades no acesso e permanência aos serviços do SUS, principalmente pela trans/travestifobia e escassez de serviços de saúde específicos. Tal situação, além de limitar o processo transexualizador, aumenta os índices de complicações pós-operatórias (ROCON et al., 2016).

Não obstante, apesar de ser considerada como tratamento padrão para disfunções pélvicas, foram poucos os relatos encontrados sobre as intervenções fisioterapêuticas após a CRS na literatura científica. Além da falta de assistência e orientações por parte dos profissionais da saúde sobre a importância de um acompanhamento contínuo a esse público, há também a ausência de interesse sobre a qualidade de vida sexual após CRS (FERREIRA; CAMPOS; FERREIRA, 2018). Tendo em vista essas dificuldades citadas acima e a escassez de pesquisas científicas, os objetivos deste estudo são: analisar as funções e as disfunções pélvicas e verificar o papel da fisioterapia pélvica após a cirurgia de afirmação de gênero em mulheres transexuais, através de uma revisão de literatura integrativa.

\section{Metodologia}

O atual estudo trata-se de uma revisão de literatura integrativa e, para a sua execução, foi realizada uma pesquisa em duas bases de dados eletrônicas, sendo estas:
Physiotherapy Evidence Database (PEDro) e U.S. National Library of Medicine National Institutes of Health (PubMed). Para a busca de dados foram utilizados os seguintes descritores: pessoas transgênero/ transgender persons, modalidades de fisioterapia/ physical therapy modalities, assoalho pélvico/ pelvic floor e transexualismo/ transexualism.

$\mathrm{Na}$ base de dados PubMed, foram realizadas três buscas utilizando os descritores em inglês: transsexualism e pelvic floor; transgender persons e pelvic floor; transgender persons e physical therapy modalities. Como estratégia de busca foi utilizado o operador booleano AND entre os descritores citados anteriormente. Já na base de dados da PEDro foi realizada apenas uma busca utilizando os descritores transgender persons e pelvic floor.

Foram excluídos estudos que abordavam assuntos, como: ducha retal, doenças como HIV, câncer de próstata e estudos que não citavam complicações pélvicas.

Os estudos foram avaliados de acordo com análise dos títulos e resumos de pesquisa, foram selecionados apenas estudos pertinentes ao tema proposto de abordagem. A pesquisa foi realizada entre março de 2020 até outubro de 2020, foi estabelecida uma data de corte incluindo apenas estudos publicados posteriormente ao ano 2010 , objetivando assim a construção de uma revisão de literatura atualizada.

\section{Resultados}

No primeiro levantamento realizado na base de dados PubMed, obteve-se um total de seis artigos e todos estavam de acordo com os critérios estabelecidos pelo atual estudo, portanto, os seis artigos foram inclusos.

No segundo levantamento realizado na base de dados PubMed, obteve-se um total de sete artigos, e novamente, todos estavam de acordo com os critérios estabelecidos pelo atual estudo e também foram inclusos.

No terceiro e último levantamento realizado na base de dados PubMed, foram encontrados um total de oito artigos. Após análise e seleção de publicações dos últimos dez anos, foram selecionados sete estudos.

$\mathrm{Na}$ base de dados PEDro foi realizado apenas um levantamento e não foi encontrado nenhum resultado.

Consequentemente, a busca supracitada obteve um total de 20 artigos que foram analisados através do título e do resumo. Seguidamente, de acordo com o objetivo do estudo, realizou-se uma análise, foi-se observado que três estudos se encontravam duplicados quando realizadas as buscas com diferentes descritores. Outros oito artigos não se enquadravam nos objetivos propostos por este estudo.

Ao final dessas etapas, foram encontrados nove artigos que se enquadravam e estavam de acordo com as propostas idealizadas. Os passos de buscas seguem no fluxograma da Figura I e os nove estudos que compõem a atual revisão sistemática apresentados no Quadro I. 
Figura I - Fluxograma de pesquisa

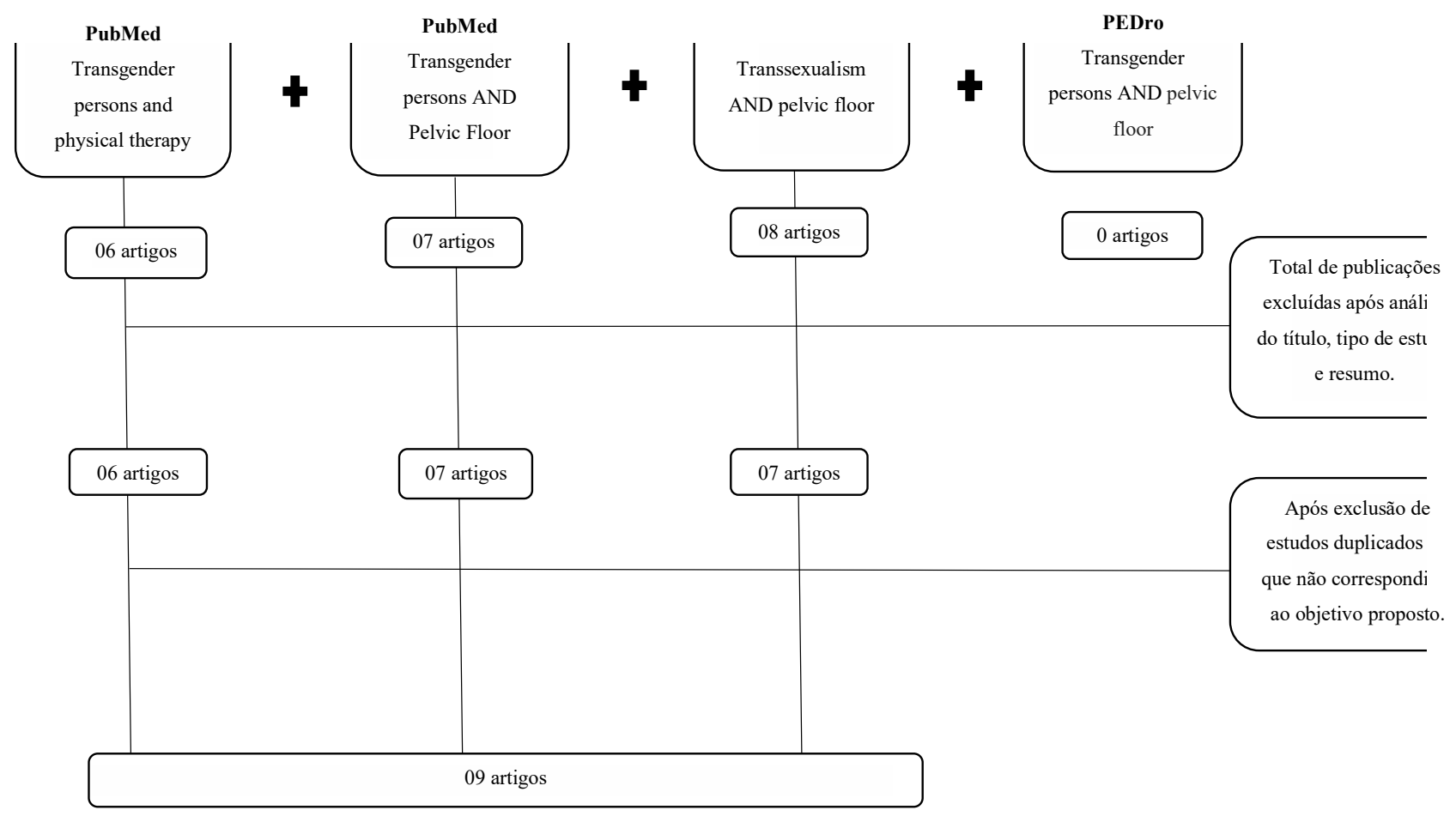

Fonte: elaborada pelas autoras. 


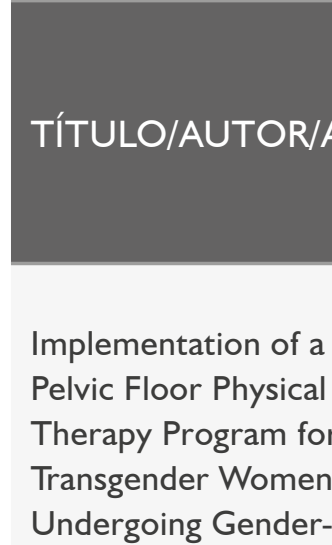

Undergoing Gender-

Affirming Vaginoplasty

DA DAVID JIANG et al.,

2019

Rev

Vaginoplasty

Complications

CECILE A. FERRANDO

2018

Revisão bibliográfica sobre as

complicações da cirurgia de

vaginoplastia, considerada como uma

técnica de cirurgia de afirmação de

gênero e as complicações clínicas sobre

essa população de pacientes.

Complications

and Patient-Reported

Outcomes in Male-to-

Female Vaginoplasty-

Where We Are Today: A

Systematic Review and

Meta-Analysis

OSCAR J. MANRIQUE,

et al., 2018

Estudo retrospectivo de análise demográficos (idade, IMC, seguro), histórico de uso de nicotina; de saúde mental; médica e cirúrgica.

Uma revisão sistemática e meta-análise com busca na PubMed, Ovid MEDLINE Epub Ahead of Print, Ovid Medline,

Ovid MEDLINE, Ovid EMBASE,

Ovid Cochrane Central Register of

Controlled Trials e Web of Science. vaginoplastia intestinal não houve

\section{DISFUNÇÕES PÉLVICAS PÓS \\ CIRURGIA DE AFIRMAÇÃO DE \\ GÊNERO}

FUNÇÕES PÉLVICAS

Foram analisados 67 prontuários

e observado: disfunção intestinal,

disfunção urinária e disfunções

musculares do assoalho pélvico (como

contração mal coordenada, relaxamento

deficiente e/ ou fraqueza muscular).

Estenose neovaginal; necrose parcial da vagina e clitóris, dor genital, lesão retal, fístula retovaginal, prolapso neovaginal, estenose meatal uretral, alteração na função miccional, incontinência urinária, deiscência da ferida, abscessos locais, hematoma, retardo na cicatrização da ferida, infecções da bexiga e sangramento pós-operatório.

Observaram em comum nas duas técnicas, fistulas, estenose, necrose do tecido, prolapso neovaginal. E na necrose tecidual.

vantagens a autolubrificação,
PÓS CIRURGIA

DE AFIRMAÇÃO

DE GÊNERO E

FISIOTERAPIA PÉLVICA

As pacientes que realizaram

\section{ATUAÇÃO DA} FISIOTERAPIA

FT pré-operatória

obtiveram taxas menores

de disfunções. As pacientes

com disfunção muscular do

assoalho pélvico e disfunção

intestinal tiveram resolução

rápida e a maioria sucesso

na dilatação após a cirurgia.

A vaginoplastia de inversão peniana permite relações

sexuais vaginais com

penetração. A vaginoplastia

intestinal tem como

obtenção de boa

profundidade neovaginal

e redução do risco de

estenose pós-operatória.

Uma taxa alta de satisfação de com resultados gerais, com resultados funcionais e com resultados estéticos.

Capacidade de ter orgasmo.

O comprimento da cavidade vaginal foi de $12,5 \mathrm{~cm}$
Fisioterapia do assoalho pélvico é benéfica no tratamento da dor pélvica e da dispareunia, e pode ajudar a tratar a incontinência urinária.

Além de ter um papel importante tanto no pré-operatório quando no pós-operatório.

Fisioterapia atua quando se tem uma hipertonicidade, espasticidade e sensibilidade, além de ruptura dos músculos que levam a disfunções do assoalho pélvico. 
(continuação)

\begin{tabular}{|c|c|c|c|c|}
\hline TÍTULO/AUTOR/ANO & $\begin{array}{l}\text { INFORMAÇÕES } \\
\text { METODOLÓGICAS }\end{array}$ & $\begin{array}{l}\text { DISFUNÇÕES PÉLVICAS PÓS } \\
\text { CIRURGIA DE AFIRMAÇÃO DE } \\
\text { GÊNERO }\end{array}$ & $\begin{array}{l}\text { FUNÇÕES PÉLVICAS } \\
\text { PÓS CIRURGIA } \\
\text { DE AFIRMAÇÃO } \\
\text { DE GÊNERO E } \\
\text { FISIOTERAPIA PÉLVICA }\end{array}$ & $\begin{array}{l}\text { ATUAÇÃO DA } \\
\text { FISIOTERAPIA }\end{array}$ \\
\hline $\begin{array}{l}\text { Quality of Life and Patient } \\
\text { Satisfaction Following } \\
\text { Male-to-Female Sex } \\
\text { Reassignment Surgery } \\
\text { NIKOLAOS A. } \\
\text { PAPADOPULOS et al., } \\
2017 .\end{array}$ & $\begin{array}{l}\text { Estudo observacional, foram } \\
\text { coletados dados sobre questões } \\
\text { demográficas, socioeconômicas } \\
\text { e satisfação pós-operatória. Foram } \\
\text { utilizados: questionário padronizado } \\
\text { de autoavaliação e qualidade de vida } \\
\text { (FLZ M). }\end{array}$ & & $\begin{array}{l}\text { A grande maioria } \\
\text { experimentou melhora na } \\
\text { QV. As pacientes declararam } \\
\text { que a feminilidade aumentou } \\
\text { consideravelmente, assim } \\
\text { como a satisfação em } \\
\text { relação a autoimagem. }\end{array}$ & \\
\hline $\begin{array}{l}\text { Patient-Reported } \\
\text { Esthetic and Functional } \\
\text { Outcomes of Primary } \\
\text { Total Laparoscopic } \\
\text { Intestinal Vaginoplasty in } \\
\text { Transgender Women With } \\
\text { Penoscrotal Hypoplasia } \\
\text { MARK-BRAM BOUMAN } \\
\text { et al., 20I6 }\end{array}$ & $\begin{array}{l}\text { Estudo observacional com aplicação } \\
\text { de questionários: Escala de Felicidade } \\
\text { Subjetiva, Escala de Satisfação com } \\
\text { a Vida, Cantril’ s Escala de Escada } \\
\text { da Vida, Índice de Função Sexual } \\
\text { Feminina, Escala de Autoimagem } \\
\text { Genital Feminina, Escala de assoalho } \\
\text { pélvico hiperativo de Amsterdam e } \\
\text { questionário sobre satisfação pós- } \\
\text { operatória. }\end{array}$ & & $\begin{array}{l}\text { A Cantril's Escala da } \\
\text { Escada da Vida e Escala } \\
\text { de Satisfação com a Vida } \\
\text { indicaram alta satisfação com } \\
\text { a vida. A funcionalidade e } \\
\text { estética foram avaliadas em } \\
\text { uma pontuação } 8,0 \text { de } 10 . \\
\text { O Índice de Função Sexual } \\
\text { Feminina de mulheres trans } \\
\text { sexualmente ativas foi de } \\
26,0 \pm 6,8 \text {. }\end{array}$ & \\
\hline $\begin{array}{l}\text { Long-Term Follow-Up } \\
\text { of Transgender Women } \\
\text { After Secondary Intestinal } \\
\text { Vaginoplasty } \\
\text { WOUTER B. VAN DER } \\
\text { SLUIS, } 2016\end{array}$ & $\begin{array}{l}\text { Estudo observacional onde observou-se } \\
\text { características cirúrgicas, complicações } \\
\text { e reoperações. } \\
\text { Instrumentos utilizados: Questionário } \\
\text { de Qualidade de Vida, Índice de Função } \\
\text { Sexual Feminina, Escala de Assoalho } \\
\text { Pélvico Hiperativo de Amsterdam } \\
\text { para Mulheres, Escala de Autoimagem } \\
\text { Genital Feminina e Questionário de } \\
\text { Autoavaliação de Vaginoplastia. } \\
\text { Foi realizado também exame físico, } \\
\text { exame endoscópico e histológico da } \\
\text { neovagina. }\end{array}$ & $\begin{array}{l}\text { Corrimento, sangramento (pós) coito, } \\
\text { dor, tenesmo, mau cheiro, intestino } \\
\text { irritável, infecções do trato urinário, } \\
\text { problemas retais, fibrose e estenose } \\
\text { neovaginal. } \\
\text { Reoperações observadas foram: } \\
\text { correção dos lábios, meatoplastia, } \\
\text { neovaginal, correção de fístula, correção } \\
\text { de prolapso e remoção cirúrgica de um } \\
\text { corpo estranho neovaginal. }\end{array}$ & $\begin{array}{l}\text { Elevada satisfação geral com } \\
\text { a vida, com relações sexuais } \\
\text { satisfatórias. Consideraram } \\
\text { sua profundidade neovaginal } \\
\text { adequada. A excitação } \\
\text { sexual foi possível nessas } \\
\text { pacientes e elas relataram } \\
\text { serem capazes de atingir } \\
\text { o orgasmo por meio da } \\
\text { estimulação direta do } \\
\text { neoclitóris. A funcionalidade } \\
\text { genital e a aparência geram } \\
\text { uma boa satisfação }\end{array}$ & \\
\hline
\end{tabular}




\begin{tabular}{|c|c|c|c|c|}
\hline TÍTULO/AUTOR/ANO & $\begin{array}{l}\text { INFORMAÇÕES } \\
\text { METODOLÓGICAS }\end{array}$ & $\begin{array}{l}\text { DISFUNÇÕES PÉLVICAS PÓS } \\
\text { CIRURGIA DE AFIRMAÇÃO DE } \\
\text { GÊNERO }\end{array}$ & $\begin{array}{l}\text { FUNÇÕES PÉLVICAS } \\
\text { PÓS CIRURGIA } \\
\text { DE AFIRMAÇÃO } \\
\text { DE GÊNERO E } \\
\text { FISIOTERAPIA PÉLVICA }\end{array}$ & $\begin{array}{l}\text { ATUAÇÃO DA } \\
\text { FISIOTERAPIA }\end{array}$ \\
\hline $\begin{array}{l}\text { Aesthetic and } \\
\text { Functional Outcomes of } \\
\text { Neovaginoplasty Using } \\
\text { Penile Skin in Male to } \\
\text { Famele Transsexuals } \\
\text { BUNCAMPER et al., } 2015\end{array}$ & $\begin{array}{l}\text { Estudo observacional com aplicação } \\
\text { do Índice de Função Sexual Feminina } \\
\text { (FSFI), uma combinação da Escala } \\
\text { de Assoalho Pélvico Hiperativo de } \\
\text { Amterdam- mulheres (AHPFS-W) } \\
\text { e a Escala de Autoimagem Genital } \\
\text { Feminina para a autoavaliação da } \\
\text { vaginoplastia. }\end{array}$ & & $\begin{array}{l}\text { A funcionalidade e a } \\
\text { aparência receberam uma } \\
\text { pontuação excelente, porém } \\
\text { para uma grande parte são } \\
\text { sexualmente disfuncionais } \\
\text { de acordo com o FSFI, } \\
\text { principalmente por não } \\
\text { serem sexualmente ativas } \\
\text { ou devido a problemas de } \\
\text { lubrificação e desconforto. }\end{array}$ & \\
\hline $\begin{array}{l}\text { Outcome of Vaginoplasty } \\
\text { in Male-to-Female } \\
\text { Transgenders: A } \\
\text { Systematic Review of } \\
\text { Surgical Techniques } \\
\text { SOPHIE ER HORBACH, } \\
\text { et al., } 2015\end{array}$ & $\begin{array}{l}\text { Revisão sistemática que objetivou } \\
\text { apresentar todas as técnicas atualmente } \\
\text { disponíveis para vaginoplastia em } \\
\text { transgêneros de homem para mulher. }\end{array}$ & $\begin{array}{l}\text { Estenose vaginal, defeitos de pele } \\
\text { transplantada, hematoma, fístula, } \\
\text { prolapso vaginal e infecção. Necrose } \\
\text { parcial da neovagina, e do clítoris, } \\
\text { perda involuntária de urina, dor } \\
\text { genital, sangramento e odor só foram } \\
\text { mencionados após a vaginoplastia } \\
\text { retossigmóide. }\end{array}$ & $\begin{array}{l}\text { As pacientes estavam tendo } \\
\text { relações sexuais (vaginais) } \\
\text { após a cirurgia, satisfeitas } \\
\text { quanto à profundidade, } \\
\text { aparência da vagina e } \\
\text { poucas se arrependem de } \\
\text { terem realizado a cirurgia } \\
\text { de afirmação de gênero, e } \\
\text { relataram a melhora na QV. }\end{array}$ & \\
\hline $\begin{array}{l}\text { Vaginal prolapse, pelvic } \\
\text { floor function, and related } \\
\text { symptoms } 16 \text { years after } \\
\text { sex reassignment surgery } \\
\text { in transsexuals } \\
\text { ANETTE KUHN, 20II }\end{array}$ & $\begin{array}{l}\text { Preencheram o SPS-Q (sintomas } \\
\text { urinários e intestinais) e foram } \\
\text { examinadas ginecologicamente. } \\
\text { O estadiamento do prolapso foi } \\
\text { realizado no pós-operatório usando } \\
\text { o ICS, POP-Q (determina a posição } \\
\text { da vagina) e o sistema de graduação } \\
\text { de Oxford para análise da contração } \\
\text { da musculatura. }\end{array}$ & $\begin{array}{l}\text { Apresentaram prolapso maior ou } \\
\text { semelhante ao ICS-POP estágio } 2 \\
\text { e necessitaram de cirurgia. } \\
\text { Sintomas urinários: dificuldades para } \\
\text { urinar, urgência, incontinência de } \\
\text { urgência e incontinência de esforço. } \\
\text { Sintomas fecais: urgência fecal e o } \\
\text { esvaziamento intestinal incompleto. } \\
\text { Além disso, relataram que nunca ficaram } \\
\text { satisfeitas com sua função sexual. }\end{array}$ & & \\
\hline
\end{tabular}

Fonte: elaborada pelas autoras. 


\section{Discussão}

A vaginoplastia de inversão peniana vem sendo a técnica mais utilizada em cirurgias de afirmação de gênero de homem para mulher, segundo Manrique e outros (2018), a inversão peniana, é a principal escolha, pois permite a formação da genitália externa feminina e a cavidade vaginal com retalhos locais. Entretanto, Buncamper e outros (20I5), observaram como vantagem o fato de a pele do pênis ser em sua maioria sem pelos, além de possuir menor probabilidade de contrair-se em comparação aos enxertos de pele de espessura, parcial ou total. No estudo de Ferrando (2018), esse procedimento sofre diversas modificações para melhorar os resultados funcionais e estéticos.

De acordo com Manrique e outros (2018), apesar de ser uma das técnicas mais utilizadas, tem como desvantagens, o uso prolongado de dilatadores, contração do canal vaginal, mau cheiro e a necessidade de lubrificação para a relação sexual. Na pesquisa de Sluis e outros (2016), a vaginoplastia de inversão peniana realmente é o principal tipo de técnica cirúrgica para mulheres trans. No entanto, esta técnica fornece profundidade neovaginal inadequada nas pacientes que não têm pele penoescrotal suficiente e em outros resultados insatisfatórios, obtém uma recomendação a vaginoplastia intestinal, técnica consagrada para reconstrução da neovagina.

Como desvantagens, tem-se o risco de cirurgia do intestino abdominal, muco excessivo e secreção fétida associada a prolapso, segundo Ferrando (2018). Para Manrique e outros (2018), a decisão do melhor procedimento deve ser adaptada para a anatomia, ao tempo de terapia hormonal, experiência do cirurgião e às necessidades da paciente. Horbach e outros (2015) concordam com essa afirmação e concluem que não é possível identificar a melhor técnica devido à falta de evidências de alta qualidade e à heterogeneidade das técnicas cirúrgicas. Inúmeras são as complicações da vaginoplastia de inversão peniana, dentre elas estão sangramento, infecção, prolapso vaginal, estenose meatal, necrose do clitóris e fibrose reto-neovaginal, conforme citado por Buncamper e outros (20I5).

Nos estudos de Ferrando (2018) e Horbach e outros (2015) há um consenso sobre as complicações supracitadas e ambos ainda listam em acréscimo: dor genital, lesão retal, necrose parcial da vagina, alterações na função miccional, incontinência urinária de esforço, urgência e mista, ruptura da parede neovaginal após a relação sexual, deiscência da ferida, abcessos locais e hematomas.

Quanto às complicações da vaginoplastia intestinal Horbach e outros (2015) citam estenose neovaginal e do introito vaginal, prolapso da mucosa, fístula retrovaginal e necrose da neovagina. Para Manrique e outros (2018), nesse tipo de vaginoplastia não ocorre a necrose tecidual. Kuhn, Santi e Birkhäuser (20I I) citam ainda o carcinoma, urgência fecal e esvaziamento incompleto do intestino.
Em Ferrando (2018), além das complicações citadas nos estudos anteriores, são incluídos secreção/mau cheiro, vazamento de uma anastomose intercólica, disfunção miccional e distúrbios na cicatrização das feridas, além de afirmar que todas essas complicações podem ser evitadas no momento da cirurgia com um planejamento pré-operatório e o uso preventivo de certas estratégias.

Segundo jiang e outros (2019), quando se fala de planejamento pré-operatório, a fisioterapia pélvica foi benéfica para dor pélvica, disfunção sexual relacionada a dor, e pode auxiliar a tratar a incontinência urinária. A fisioterapia pré-operatória pode identificar qualquer limitação ou incapacidade funcional, analisar os padrões basais da micção e da defecação, além de avaliar as condições do assoalho pélvico. No pós-operatório a fisioterapia pélvica pode contribuir na dilatação da neovagina e melhora funcional dos músculos do assoalho pélvico hipoativos.

Além disso, de acordo com Jiang e outros (2019), o fisioterapeuta pode educar os pacientes sobre a função e a anatomia do assoalho e fornecer conselhos práticos sobre a dilatação, como o posicionamento do corpo para facilitar o relaxamento e a abertura da saída óssea pélvica. No mesmo estudo foram orientados exercícios domiciliares, incluindo exercícios respiratórios com contração coordenada do assoalho pélvico e alongamentos da região lombo-pélvica que auxiliam na obtenção das posições necessárias para a inserção ativa do dilatador.

Em contrapartida, para Ferrando (20I8), a fisioterapia só se torna útil nos casos de ruptura da musculatura, levando a disfunções do assoalho pélvico, que desencadeia uma dificuldade de esvaziar a bexiga, sintomas urinários e miccionais. Além de quando revelada uma hipertonicidade, espasticidade e sensibilidade. $\mathrm{Na}$ pesquisa de Horbach e outros (20I5), o resultado foi conflitante, pois alguns pacientes referiram dispaurenia, anorgasmia e sangramento neovaginal, enquanto outras se apresentaram satisfeitas e felizes com a função sexual. Neste estudo foi observada satisfação com a profundidade vaginal.

Horbach e outros (2015) afirmam ainda que a maioria das mulheres trans ficam satisfeitas com a aparência estética e a minoria não fica satisfeita principalmente com os grandes lábios ou com os pequenos lábios. Já Buncamper e outros (20I5), afirmam que nunca foi realmente avaliado se mulheres trans estão satisfeitas com o resultado, principalmente com a cirurgia corretiva, apesar de a maioria se mostrar bastante satisfeita. As que permanecem infelizes é por conta da falta de incapacidade de penetração, falta de sensação prazerosa e outras razões desconhecidas.

No estudo de Papadopulos e outros (2017), em concordância com Sluis e outros (2016), os níveis de satisfação com os resultados estéticos foram muito bons. Tanto na vaginoplastia intestinal quanto na vaginoplastia de inversão peniana, os autores concluem que é possível se 
ter relação sexual e chegar a um orgasmo e que a satisfação com a vida aumenta, chegando até a gerar uma maior felicidade. Para Kuhn, Santi e Birkhäuser (20II), a maioria das pacientes relatam que a vida sexual interfere em algum grau com o prazer com a vida para melhor ou para pior e, algumas nunca ficaram satisfeitas com a função sexual, uma das causas dessa insatisfação é o prolapso. Já para Bouman e outros (2016), a maioria das mulheres se apresentam satisfeitas, porém aquelas que relatam insatisfação, geralmente é devido à assimetria dos grandes lábios.

Segundo Ferrando (2018), a felicidade autorrelatada pelas pacientes por terem feito a cirurgia de afirmação de gênero não foi associada à ausência ou presença desses sintomas e, de maneira geral, a maioria dos pacientes ficam felizes com a cirurgia.

\section{Considerações finais}

Observou-se que as cirurgias de afirmações de gênero, independente da técnica escolhida, pode apresentar complicações pélvicas, urinárias, intestinais e/ou sexuais além de algias. Porém, os estudos apontam que o exercício da sexualidade positiva, a função sexual prazerosa e a feminilidade das mulheres transexuais são os principais ganhos após procedimentos cirúrgicos. Existe uma escassez de artigos na literatura sobre a atuação da fisioterapia frente a esse perfil de pacientes, com citação dos protocolos e técnicas utilizadas. Portanto, mais estudos com qualidade metodológica devem ser realizados nessa temática. As autoras declaram que não houve financiamento para este trabalho e nenhum conflito de interesse.

\section{Referências}

\section{AMERICAN PSYCHOLOGICAL ASSOCIATION.}

Guidelines for psychological practice with transgender and gender nonconforming people. American Psychologist, Washington, DC, v. 70, n. 9, p. 832-864, 2015 Disponível em: https:// content.apa.org/record/20I5-55237-002. Acesso: 09 Jan. 2021.

BOUMAN, M. B. et al. Patient-Reported Esthetic and Functional Outcomes of Primary Total Laparoscopic Intestinal Vaginoplasty in Transgender Woman With Penoscrotal Hypoplasia. The Journal of Sexual Medicine, Amsterdam, v. I3, n. 9, p. I438-I444, 2016. Disponível em: https://linkinghub.elsevier.com/ retrieve/pii/SI 743-6095(I6)30300-9. Acesso: 20 Sept. 2020.
BUNCAMPER, M. E. et al. Aesthetic and Functional Outcomes of Neovaginoplasty Ursing Penile Skin in Male-to-Female Transsexuals. The Journal of Sexual Medicine, Malden, v. 12, n. 7, p. 1626-1634, 2015. Disponível em: https://www. ncbi.nlm.nih.gov/pmc/articles/PMC33 I 2 I 44/. Acesso em: 2 nov. 2020.

\section{CIASCA, S.; HERCOWITZ, A.; LOPES}

JUNIOR, A. Saúde LGBTQIA+Práticas de cuidado transdisciplinar. Campo Belo: Manole, 202I.

FERRANDO, C. A. Vaginoplasty Complications. Clinics in Plastic Surgery, Philadelphia, v. 45, n. 3, p. 36I-368, 20I8. Disponível em: https:// www.researchgate.net/publication/324I 25738 Vaginoplasty_Complications. Acesso em: 18 out. 2020.

FERREIRA, B. R. A.; SILVA, F. J. D. S. C. A intervenção fisioterapêutica na reabilitação pós cirurgia de redesignação de sexo masculino para feminino: relato de caso. Revista Pesquisa em Fisioterapia, Salvador, v. I0, n. 2, p. 288-300, 2020. Disponível em: https://www5.bahiana. edu.br/index.php/fisioterapia/article/view/2854. Acesso em: 18 maio 2020.

FERREIRA, M. C. S.; CAMPOS, S. R.; FERREIRA, A. P. M. Repercussões da redesignação sexual masculino para feminino e a atuação da fisioterapia. E-Scientia, Belo Horizonte, v. II, n. 2, 2018. Disponível em: https://revistas.unibh. br/dcbas/article/view/2402. Acesso: 20 jun. 2020.

GALVÃO, M. H. et al. Fisioterapia no pósoperatório de cirurgia de redesignação de sexo: estudo de caso. In: CONGRESSO BRASILEIRO DE FISIOTERAPIA NA SAÚDE DA MULHER, 4., 2018. Belo Horizonte. Anais [...]. Belo Horizonte: Abrafism; Cobraf, 2018. v. 2, n. I. Disponível em: http://www.anaiscobraf.com.br/arqAnais/ Fisioterapia_no_posoperatorio_de_cirurgia_de_ redesignacao_de_sexo.pdf. Acesso: 8 jun. 2020.

HORBACH, S. E. et al. Outcome of Vaginoplasty in Male-to-Female Transgenders: A Systematic Review of Surgical Techniques. The Journal of Sexual Medicine, Malden, v. I2, n. 6, p. 149915I2, 20I5. Disponível em: https://linkinghub. elsevier.com/retrieve/pii/SI 743-6095(I 5)3 I 059-6 Acesso em: 15 out. 2020. 
JESUS, J. G. Orientações sobre identidade de gênero: conceitos e termos. 2. ed. Brasília, DF: o autor, 20I2. Disponível em: http:// www.diversidadesexual.com.br/wp-content/ uploads/20I3/04/G\%C3\%8ANERO-CONCEITOSE-TERMOS.pdf. Acesso: 6 ago. 2020.

JIANG, D. D. et al. Implementation of a Pelvic Floor Physical Therapy Program for Transgender Women Undergoing GenderAffirming Vaginoplasty. Obstetrics \& Gynecology, Hagerstown, v. I33, n. 5, p. I003-10II, 2019. Disponível em: https://journals.Iww. com/greenjournal/Abstract/2019/05000/ Implementation_of_a_Pelvic_Floor_Physical_ Therapy.22.aspx. Acesso em: 20 set. 2020.

JOSEPH, A. et al. Gender identity and the management of the transgender patient: a guide for non-specialists. Journal of the Royal Society of Medicine, London, v. II 0, n. 4 p. I44-I52, 2017. Disponível em: https://journals.sagepub.com/ doi/I0. I I77/0 I 4 I0768I7696054. Acesso: 19 dez. 2020.

KUHN, A.; SANTI, A.; BIRKHÄUSER, M. Vaginal prolapse, pelvic floor function, and related symptons 16 years after sex reassignment surgery in transsexuals. Fertility and Sterility, New York, v. 95, n. 7, p. 2379-2382, 20I I. Disponível em: https://www.fertstert.org/article/SOOI50282(I I)00438-9/fulltext. Acesso: 20 set. 2020.

MANRIQUE, O. J. et al. Complications and Patient-Reported Outcomes in Male-toFamele Vaginoplasty - Where We Are Today: A Systematic Review and Meta-Analysis. Annals of Plastic Surgery, Boston, v. 80, n. 6 , p. 684-69I, 2018. Disponível em: https:// journals.Iww.com/annalsplasticsurgery/ Abstract/2018/06000/Complications_and_ Patient_Reported_Outcomes_in.19.aspx. Acesso em: 21 set. 2020.

PAPADOPULOS, N. A. et al. Quality of Life and Patient Satisfaction Followng Male-to-Female Sex Reassignment Surgery. The Journal of Sexual Medicine, Amsterdam, v. 14, n. 5, p. 721-730, 2017. Disponível em: https://linkinghub.elsevier. com/retrieve/pii/SI 7436095I 730 I 224. Acesso em: 2I Sept. 2020.
ROCON, P. C.; SODRE, F.; RODRIGUES,

A. Regulamentação da vida no processo transexualizador brasileiro: uma análise sobre a política pública. Revista Katálysis, Florianópolis, v. 19, n. 2, p. 260-269, jul./set. 2016. Disponível em: https://www.scielo.br/scielo.php?pid=SI4 I4$49802016000200260 \&$ script $=$ sci

abstract\&tlng=pt. Acesso: maio 2020 .

ROCON, P. C. et al. Dificuldades vividas por pessoas trans no acesso ao Sistema Único de Saúde. Ciência \& Saúde Coletiva, Rio de janeiro, v. 21 , n. 8, p. 25I7-2526, ago. 2016. Disponível em: https://www.scielo.br/scielo.php?script $=$ sci arttext\&pid $=S|4| 3-8|2320| 60008025 \mid 7$ Acesso: 9 maio 2020.

SOUSA, E. C. de et al. Complicações operatórias na cirurgia de redesignação sexual: uma revista sistemática. Brazilian Journal of Health Review, Curitiba, v. 2, n. 3, p. 1624-1632, mar./abr. 2019. Disponível em: https://www. brazilianjournals.com/index.php/BJHR/article/ view/I4I5\#: : :text $=$ Entre\%20as\%20 complica\%C3\%A7\%C3\%B5es\%20mais\%20 comuns, necrose \%20tecidual\%20s\%C3\%A30\% 20complica\%C3\%A7\%C3\%B5es\%20descritas. Acesso em: 8 maio 2020.

VAN DER SLUIS, W. B. et al. Long-Term FollowUp of Transgender Woman After Secondary Intestinal Vaginoplasty. The Journal of Sexual Medicine, Amsterdam, v. 13, n. 4, p. 702-7I0, 2016. Disponível em: https://linkinghub.elsevier. com/retrieve/pii/SI 743-6095(I6)00085-0. Acesso em: 22 set. 2020.

VENTRIGLIO, A.; BHUGRA, D. Sexuality in the 2 Ist Century: Sexual Fluidity. East Asian Archives of Psychiatry, Hong Kong, v. 29, n. I, p. 30-34, 2019. Disponível em: https://www.easap.asia/ index.php/find-issues/current-issue/item/834| 903-v29n I-p30. Acesso em: 09 Jan 202।.

VIEIRA, et al. Lidando com a função sexual de mulher no ciclo gravídico-puerperal. Rio de Janeiro: Federação Brasileira das Associações de Ginecologia e Obstetrícia, 2017. 